\title{
Novel clinical biomarkers in blood and pleural effusion for diagnosing patients with tuberculosis distinguishing from malignant tumor
}

Jian Wang

Macau University of Science and Technology, Macau (SAR)

\section{Zhe-Xiang Feng}

Hubei University of Medicine

Tao Ren

Hubei University of Medicine

Wei-Yu Meng

Macau University of Science and Technology, Macau (SAR)

Imran Khan

Macau University of Science and Technology, Macau (SAR)

Xing-Xing Fan

Macau University of Science and Technology, Macau (SAR)

Hu-Dan Pan

Macau University of Science and Technology, Macau (SAR)

Liang Liu

Macau University of Science and Technology, Macau (SAR)

Yi-Jun Tang

Hubei University of Medicine

Xiao-Jun Yao

Macau University of Science and Technology, Macau (SAR)

Run-Ze Li

Macau University of Science and Technology, Macau (SAR)

Mei-Fang Wang

Hubei University of Medicine

Elaine Lai-Han Leung ( $\nabla$ lhleung@must.edu.mo)

Macau University of Science and Technology, Macau (SAR)

\section{Research Article}

Keywords: pleural effusion, tuberculous, cancerous, biomarker, diagnosis 
Posted Date: November 16th, 2021

DOI: https://doi.org/10.21203/rs.3.rs-1049353/v1

License: (c) (i) This work is licensed under a Creative Commons Attribution 4.0 International License. Read Full License 


\section{Abstract \\ Background}

Pleural effusion (PE) is a common manifestation of tuberculosis and malignant tumors, but it is difficult to distinguish tuberculous pleural effusion (TPE) and malignant pleural effusion (MPE), especially by non-invasive detection indicators. We aimed to find effective detection indexes in blood and PE for differentiating patients with tuberculosis from a malignant tumor.

\section{Methods}

815 patients were collected who diagnosed with tuberculosis or cancer at Taihe hospital from 2014 to 2017. 717 patients were found to have PE by thoracoscopy. The clinical characteristics, patients' blood parameters, and PE indicators information were summarized for analysis.

\section{Results}

The patients with MPE had higher percentages to be bloody and negative of Rivalta test in PE than patients with TPE. Then for clinical indicators, comparing specific parameters in blood, we observed 18 indicators were higher in the TPE group than in the MPE group. On contrast, 12 indicators were higher in the MPE group than in the TPE group $(p<0.01)$. In addition, in PE tests, we found there were 3 parameters higher in TPE and other 4 parameters higher in MPE patients group $(p<0.01)$. Then for clinical diagnosing practice, ROC and PCA analysis were applied. Top six relevant indicators with AUC value over 0.70 were screened out: pADA (0.90), pHsCRP (0.79), sMONp (0.75), sHsCRP (0.73), sESR (0.71), and sDdimer (0.70). Moreover, with the Logistic regression model, a specific combination of 3 biomarkers pADA, sMONp, and sHsCRP could enhance distinguishing tuberculosis from malignant tumor patients with PE (AUC=0.944, 95\% $\mathrm{Cl}=0.925-0.964)$. For the top single marker pADA, we further analyzed its diagnostic function in patients with different group and observed it kept the high specificity and sensitivity.

\section{Conclusions}

The six indicators of pADA, pHsCRP, sMONp, sHsCRP, sESR and sD-dimer showed significant diagnostic value for clinicians. Further, the combination of pADA, sMONp, and sHsCRP has high accuracy for differential diagnosis for the first time. Mostly interestingly, pADA single marker maintained high specificity and sensitivity in patients with different status, which has great value for the rapid and accurate diagnosis of suspected cases.

\section{Background}


Pleural effusion (PE) is mainly seen in various types of inflammation, tuberculosis, and malignant tumors [1-3]. The onset of tuberculous PE is more insidious [4], with a slow course and lack specificity [5]. Malignant PE is also common, with about $20 \%$ being the first symptom and $30-40 \%$ occurring in the course of the disease, indicating poor prognosis and short survival[6]. Patients with MPE had a worse prognosis compared to patients without MPE (median survival of 7.49 vs 12.65 months, $p<0.001$ ) [6]. Early diagnosis and treatment can lead to a better prognosis.

In the current clinical practice, isolation of mycobacterium tuberculosis in the pleural fluid is difficult and can be negative in the acute setting[7]. However, the main obstacle in diagnosing malignant effusions is the presence of false-negative cytological results in about $40 \%$ of cases[8]. More invasive procedures (eg, a pleural biopsy) to identify caseating granuloma from the parietal pleura may be required.

Thoracoscopic surgery is decisive for TPE and MPE[9], but it is not widely used because of its invasive property. Consequently, the development of noninvasive methods is important to differentially diagnose these two diseases.

Currently, there has been reported some non-invasive studies to identify patients with PE of tuberculosis from malignant tumor [10-12], such as serum-total protein, albumin, globulin could be significantly higher in tuberculosis(TB) group than lung cancer group, at the same time, serum lactate dehydrogenase(sLDH) higher in lung cancer group than in TB group $(p<0.01)$ [13]. Some researchers found that the serum D-dimer level of TPE patients was higher than MPE patients[14]. It also has been proved that lymphocytes and macrophages were the predominant nucleated cell in MPE, TPE was characterized by a large percentage of leukocytes and lymphocytes $(p<0.01)$ [15]. However, these results all meet the problems of low sensitivity and low specificity. In this study, we investigated clinical data of 717 patients with TPE or MPE, analyzing their clinical characteristics, hydrothorax parameters, and blood parameters. This study has a large sample size. At the same time, significant indicators in the differential diagnosis are preliminarily expounded, which is beneficial to distinguish PE early and improve the accuracy of diagnosis.

\section{Methods}

\subsection{Data collection}

A total of 815 patients diagnosed with tuberculosis or cancer from Taihe hospital, between 2014 and 2017, and were recruited in this study. All patients agreed to participate in the study and signed informed consent forms. All patients underwent thoracoscopic examination, 717 patients had PE. In the tuberculosis group, there were 641 cases, including 453 males and 188 females, aged 15-90 years. Aged<25 years 149 cases, aged 25-45 years 245 cases, aged $45-65$ years 191 cases, aged $>65$ years 56 cases. There were 570 patients with PE. In the cancer group, there were 174 cases, including 92 males and 82 females, aged 15-90 years. Aged<25 years 1 case, aged $25-45$ years 15 cases, aged $45-65$ years 102 cases, aged $>65$ years 56 cases. There were 147 patients with PE. 
Inclusion criteria for tuberculous PE [16]: Pathological examination revealed tuberculosis foci; Positive for acid-fast staining or positive for the culture of mycobacterium tuberculosis, or significant absorption of $\mathrm{PE}$ in anti-tuberculosis treatment; At least one of the above criteria should be met. Inclusion criteria for cancerous PE [17]: imaging examination showed thoracic mass shadow; PE was exudative; negative for acid-fast staining or negative for tuberculosis bacillus culture; The histological or cytological examination confirmed malignant tumor; All the above criteria must be met.

\subsection{Pe And Blood Statistical Analysis}

All data below were collected from Taihe hospital.

PE analysis indicators include: pADA(hydrothorax adenosine dehydrogenase), pAMS(hydrothorax amylase), pCell(hydrothorax cells), pGLU(hydrothorax glucose), pHsCRP(hydrothorax high sensitivity C reactive protein), pMON(hydrothorax monocytes), pNC(hydrothorax nucleated cells), pTC(hydrothorax total cholesterol), pTP(hydrothorax total protein);

Serum analysis indicators include: sAlb(blood albumin), sALP(blood alkaline phosphatase), sALT(alanine aminotransferase), sAPTT(activated partial prothrombin time), SAST(aspartate aminotransferase), sA/G(blood A/G), sBASp(percentage of blood basophil cells), sCa(serum calcium), sCK(blood creatine kinase), sCKI(blood creatine kinase isoenzyme), sCL(blood chlorine), sCRE(serum creatinine), sDdimer(blood D-dimer), sEOSp(percentage of eosinophil), sESR(erythrocyte sedimentation rate), sFbg(blood fibrin), sFDP(fibrinogen degradation products), sGLO(globin), sGRANp(percentage of blood granulocytes), sHCO3-(blood bicarbonate), sHGB(hemoglobin), sHsCRP(blood high sensitivity $\mathrm{C}$ reactive protein), sINR(blood internationalization standardized ratio), sK(Serum kalium), sLDH(serum lactate dehydrogenase), sLYMp(lymphocyte percentage) sMg(serum magnesium), sMONp(percentage of blood monocyte), sNA(blood natrium), sP(serum phosphate), sPA(blood prealbumin), sPLT(platelet), sPT(prothrombin time), sPTA(prothrombin activity), sRBC(red blood cell), sPTR(prothrombin time ratio), sTB(total bilirubin), sTBA(total bile acid), sTP(blood total protein), sTT(blood thrombin time), sUREA(blood urea), sWBC(white blood cell), saHDH(blood a hydroxybutyrate dehydrogenase), sүGT(blood y glutamyl transpeptidase).

Test results using the numerical analysis, data obey the normal distribution, using SPSS18.0 software for statistical analysis of count data by chi-square test, measurement data using independent t-test, comparison with the analysis of variance between groups, ROC curve to determine the best threshold (cut off) and the AUC, after the series-parallel experiment, $p<0.05$ difference is statistically significant.

\section{Results}

\subsection{Demographic and clinical characteristics of the study population}


A total of 717 patients with TPE or MPE were recruited in this study. These patients were divided into two groups: 147 MPE patients (20.4\%) and 570 TPE patients (79.6\%) $\bigotimes$ To distinguish TPE and MPE, as shown in Table 1, we analyzed multiple clinical status of patients and physical characteristics of the patient's pleural effusion. Among patients with hydrothorax, tuberculosis patients had a higher possibility to get a fever. Besides, malignant tumor patients $(40.0 \%)$ were more likely to get bloody PE compared to tuberculosis patients. Moreover, the PE of cancer patients (67.3\%) had a higher percentage to be turbid. What's more, among patients with hydrothorax, tuberculosis patients $(91.6 \%)$ in the Rivalta test had a higher percentage to be positive (detailed data listed in Table 1).

Table 1

Demographic and clinical characteristics of the study population

\begin{tabular}{|lllll|}
\hline Features & \multicolumn{2}{l}{ Clinical diagnosis } & F/X \\
\cline { 2 - 5 } & Tuberculosis & Cancer & Total & p-value \\
\hline Hydrothorax & 641 & 174 & 815 & \\
\hline Yes & $570(88.9 \%)$ & $147(84.5 \%)$ & 717 & 2.551 \\
\hline No & $71(11.1 \%)$ & $27(15.5 \%)$ & 98 & 0.110 \\
\hline Fever & 570 & 147 & 717 & \\
\hline Yes & $346(60.7 \%)$ & $18(12.2 \%)$ & 364 & 109.79 \\
\hline No & $224(39.3 \%)$ & $129(87.8 \%)$ & 353 & 0.0001 \\
\hline Total Color of pleural effusion & 560 & 145 & $705(12$ & \\
\hline Yellow & & & missed) & 73.21 \\
\hline Pink & $471(84.1 \%)$ & $79(54.5 \%)$ & 550 & 0.0001 \\
\hline Red & $30(5.4 \%)$ & $8(5.5 \%)$ & 38 & \\
\hline Total transparency of pleural effusion & 569 & $58(40.0 \%)$ & 117 & 0.002 \\
\hline Turbid & $59(10.5 \%)$ & 147 & 716 & 18.52 \\
\hline Light turbid & $270(47.5 \%)$ & $99(67.3 \%)$ & 550 & 0.0001 \\
\hline Transparent & $182(32.0 \%)$ & $29(19.7 \%)$ & 38 & \\
\hline Rivalta test & $117(20.6 \%)$ & $19(12.9 \%)$ & 117 & $716(1 \mathrm{missed})$ \\
\hline Negative & 570 & 146 & 73 & 643 \\
\hline Positive & $48(8.4 \%)$ & $25(17.1 \%)$ & $121(82.9 \%)$ & \\
\hline
\end{tabular}




\subsection{Discriminative indicators in clinical practice to identify TPE and MPE}

To observe the clinical features of TPE and MPE, indicators in the blood and PE samples were examined. Firstly, tuberculosis and tumor patients were separated by using unsupervised hierarchical clustering with heatmap shown in Figure 1. We observed serum and PE indicators of tumor patients compared to tuberculosis patients are quite different (Figure 1).

To further find effective identification indicators, we compared and analyzed blood parameters and PE indicators through Mann-Whitney $U$ test (as shown in Table 2 and Figure 2, 37 indicators among 55 indicators between each group was statistically significant, $p<0.05)$. In the serum, the sESR, sMONp, sHsCRP and other 16 indicators were higher in the TPE group than in the MPE group (sESR: $46.27 \pm 1.16$ vs $29.78 \pm 2.32$, sMONp: $10.35 \pm 0.17$ vs $7.18 \pm 0.20$, sHsCRP: $50.30 \pm 2.67$ vs $19.25 \pm 3.20 ; p<0.01$ ). Moreover, the sWBC, sA/G and other 10 indicators were higher in the MPE group than in the TPE group (sWBC: $7.70 \pm 0.24$ vs $6.48 \pm 0.11$, sA/G: $1.49 \pm 0.08$ vs $1.19 \pm 0.02 ; p<0.01)$. Among the indicators of pleural effusion, the pADA, pHsCRP and pMON were higher in the TPE group than in the MPE group (pADA: $44.269 \pm 0.997$ vs $11.902 \pm 0.969$, pHsCRP: $24.63 \pm 1.16$ vs $8.07 \pm 0.87$, pMON: $79.66 \pm 0.94$ vs $75.33 \pm 1.74 ; p$ $<0.01$ ), the pAMS and 3 other markers was higher in the MPE group than in the TPE group(345.851 \pm 79.170 vs $40.725 \pm 1.023, p<0.01$ ) (Figure 2 , Table 2 ).

Table 2 Summary of indicators from comparative analysis of serum and pleural effusion 


\begin{tabular}{|c|c|c|c|c|c|}
\hline Indicators & Diagnosis & $\mathrm{N}$ & Mean & SEM & Mann-Whitney U Test(Sig.) \\
\hline \multirow[t]{2}{*}{ pCell } & TB & 567 & 19858.46 & 3463.52 & \multirow[t]{2}{*}{$<0.001$} \\
\hline & $\mathrm{CA}$ & 144 & 59114.03 & 11069.29 & \\
\hline \multirow[t]{2}{*}{ pMON } & TB & 563 & 79.66 & 0.94 & \multirow[t]{2}{*}{$<0.001$} \\
\hline & $\mathrm{CA}$ & 147 & 75.33 & 1.74 & \\
\hline \multirow[t]{2}{*}{ pTP } & TB & 547 & 47.99 & 0.79 & \multirow[t]{2}{*}{$<0.001$} \\
\hline & $\mathrm{CA}$ & 142 & 50.44 & 6.93 & \\
\hline \multirow[t]{2}{*}{ pGLU } & TB & 548 & 5.05 & 0.16 & \multirow[t]{2}{*}{0.001} \\
\hline & $\mathrm{CA}$ & 142 & 5.59 & 0.26 & \\
\hline \multirow[t]{2}{*}{ pAMS } & TB & 546 & 40.73 & 1.02 & \multirow[t]{2}{*}{$<0.001$} \\
\hline & $\mathrm{CA}$ & 142 & 345.85 & 79.17 & \\
\hline \multirow[t]{2}{*}{ pHsCRP } & TB & 548 & 24.63 & 1.16 & \multirow[t]{2}{*}{$<0.001$} \\
\hline & $\mathrm{CA}$ & 142 & 8.07 & 0.87 & \\
\hline \multirow[t]{2}{*}{ pADA } & TB & 547 & 44.27 & 1.00 & \multirow[t]{2}{*}{$<0.001$} \\
\hline & CA & 141 & 11.90 & 0.97 & \\
\hline \multirow[t]{2}{*}{ sPTA } & TB & 450 & 102.62 & 4.66 & \multirow[t]{2}{*}{$<0.001$} \\
\hline & CA & 121 & 114.07 & 8.63 & \\
\hline \multirow[t]{2}{*}{ sPT } & TB & 537 & 13.19 & 1.31 & \multirow[t]{2}{*}{$<0.001$} \\
\hline & $\mathrm{CA}$ & 151 & 11.78 & 0.66 & \\
\hline \multirow[t]{2}{*}{ sPTR } & $\mathrm{TB}$ & 523 & 1.46 & 0.38 & \multirow[t]{2}{*}{$<0.001$} \\
\hline & $\mathrm{CA}$ & 146 & 0.99 & 0.01 & \\
\hline \multirow[t]{2}{*}{ sINR } & TB & 537 & 1.72 & 0.36 & \multirow[t]{2}{*}{$<0.001$} \\
\hline & $\mathrm{CA}$ & 151 & 1.26 & 0.24 & \\
\hline \multirow[t]{2}{*}{ sAPTT } & TB & 537 & 31.81 & 0.25 & \multirow[t]{2}{*}{0.013} \\
\hline & $\mathrm{CA}$ & 152 & 30.95 & 0.53 & \\
\hline \multirow[t]{2}{*}{ sFbg } & $\mathrm{TB}$ & 537 & 5.44 & 0.08 & \multirow[t]{2}{*}{$<0.001$} \\
\hline & $\mathrm{CA}$ & 152 & 4.77 & 0.14 & \\
\hline \multirow[t]{2}{*}{ sD-dimer } & $\mathrm{TB}$ & 490 & 2.69 & 1.19 & \multirow[t]{2}{*}{$<0.001$} \\
\hline & CA & 141 & 0.67 & 0.07 & \\
\hline
\end{tabular}




\begin{tabular}{|c|c|c|c|c|c|}
\hline \multirow[t]{2}{*}{ sFDP } & TB & 490 & 9.45 & 0.42 & \multirow[t]{2}{*}{$<0.001$} \\
\hline & $\mathrm{CA}$ & 141 & 7.17 & 0.94 & \\
\hline \multirow[t]{2}{*}{ sESR } & TB & 530 & 46.25 & 1.16 & \multirow[t]{2}{*}{$<0.001$} \\
\hline & $\mathrm{CA}$ & 136 & 29.78 & 2.32 & \\
\hline \multirow[t]{2}{*}{ sWBC } & TB & 545 & 6.48 & 0.11 & \multirow[t]{2}{*}{$<0.001$} \\
\hline & $\mathrm{CA}$ & 155 & 7.70 & 0.24 & \\
\hline \multirow[t]{2}{*}{ sLYMp } & $\mathrm{TB}$ & 545 & 19.14 & 0.45 & \multirow[t]{2}{*}{0.037} \\
\hline & $\mathrm{CA}$ & 155 & 20.16 & 0.64 & \\
\hline \multirow[t]{2}{*}{ sEOSp } & $\mathrm{TB}$ & 545 & 2.24 & 0.22 & \multirow[t]{2}{*}{0.003} \\
\hline & $\mathrm{CA}$ & 155 & 3.37 & 0.94 & \\
\hline \multirow[t]{2}{*}{ sMONp } & TB & 545 & 10.35 & 0.17 & \multirow[t]{2}{*}{$<0.001$} \\
\hline & $\mathrm{CA}$ & 155 & 7.18 & 0.20 & \\
\hline \multirow[t]{2}{*}{ sHGB } & TB & 545 & 120.09 & 0.84 & \multirow[t]{2}{*}{0.037} \\
\hline & $\mathrm{CA}$ & 155 & 123.27 & 1.55 & \\
\hline \multirow[t]{2}{*}{ sPLT } & TB & 545 & 292.18 & 4.25 & \multirow[t]{2}{*}{$<0.001$} \\
\hline & $\mathrm{CA}$ & 155 & 255.16 & 6.38 & \\
\hline \multirow[t]{2}{*}{ sNA } & $\mathrm{TB}$ & 492 & 139.38 & 0.32 & \multirow[t]{2}{*}{$<0.001$} \\
\hline & $\mathrm{CA}$ & 127 & 140.74 & 0.37 & \\
\hline \multirow[t]{2}{*}{$s C L$} & $\mathrm{~TB}$ & 491 & 104.42 & 1.85 & \multirow[t]{2}{*}{0.001} \\
\hline & $\mathrm{CA}$ & 127 & 103.39 & 0.40 & \\
\hline \multirow[t]{2}{*}{ sALT } & $\mathrm{TB}$ & 512 & 23.65 & 1.28 & \multirow[t]{2}{*}{0.029} \\
\hline & $\mathrm{CA}$ & 123 & 15.82 & 1.14 & \\
\hline \multirow[t]{2}{*}{ sY-GT } & TB & 512 & 42.48 & 2.30 & \multirow[t]{2}{*}{0.001} \\
\hline & $\mathrm{CA}$ & 123 & 29.89 & 2.68 & \\
\hline \multirow[t]{2}{*}{ sTP } & TB & 425 & 69.07 & 1.72 & \multirow[t]{2}{*}{$<0.001$} \\
\hline & $\mathrm{CA}$ & 102 & 64.01 & 0.67 & \\
\hline \multirow[t]{2}{*}{ sGLO } & TB & 423 & 32.46 & 0.87 & \multirow[t]{2}{*}{$<0.001$} \\
\hline & $\mathrm{CA}$ & 102 & 27.26 & 0.73 & \\
\hline $\mathrm{sA} / \mathrm{G}$ & TB & 423 & 1.19 & 0.02 & $<0.001$ \\
\hline
\end{tabular}




\begin{tabular}{|c|c|c|c|c|c|}
\hline & CA & 102 & 1.49 & 0.08 & \\
\hline \multirow[t]{2}{*}{ sTB } & TB & 426 & 10.37 & 0.28 & \multirow[t]{2}{*}{$<0.001$} \\
\hline & CA & 104 & 12.38 & 0.62 & \\
\hline \multirow[t]{2}{*}{ sTBA } & TB & 454 & 4.00 & 0.27 & \multirow[t]{2}{*}{0.001} \\
\hline & CA & 106 & 2.74 & 0.24 & \\
\hline \multirow[t]{2}{*}{ SPA } & TB & 437 & 148.70 & 3.70 & \multirow[t]{2}{*}{$<0.001$} \\
\hline & CA & 102 & 193.11 & 7.51 & \\
\hline \multirow[t]{2}{*}{ sUREA } & TB & 495 & 4.69 & 0.70 & \multirow[t]{2}{*}{$<0.001$} \\
\hline & CA & 127 & 6.75 & 1.89 & \\
\hline \multirow[t]{2}{*}{ sCRE } & TB & 494 & 85.45 & 0.75 & \multirow[t]{2}{*}{0.028} \\
\hline & CA & 127 & 83.70 & 1.56 & \\
\hline \multirow[t]{2}{*}{ sCKI } & TB & 58 & 6.33 & 0.35 & \multirow[t]{2}{*}{0.038} \\
\hline & CA & 24 & 14.13 & 5.26 & \\
\hline \multirow[t]{2}{*}{ sLDH } & TB & 61 & 170.62 & 6.02 & \multirow[t]{2}{*}{0.024} \\
\hline & CA & 24 & 221.54 & 31.01 & \\
\hline \multirow[t]{2}{*}{ sHsCRP } & TB & 301 & 50.30 & 2.67 & \multirow[t]{2}{*}{$<0.001$} \\
\hline & CA & 70 & 19.25 & 3.20 & \\
\hline
\end{tabular}

\subsection{Effective markers to distinguish tuberculosis and malignant tumor with pleural effusion}

To further screen effective diagnostic indicators, these 37 indicators were applied to construct ROC Curves. Based on the AUC (area under the ROC curve value, sensitivity and specificity), top 6 indicators with higher diagnostic value were screened out (AUC $\geq 0.700$ ) (pADA, pHsCRP, sMONp, sHsCRP, sESR and sD-dimer) (Figure 3, Table 3). In addition, PCA (principal component analysis) with these 6 serum or PE indicators revealed a clear separation between tuberculosis and malignant tumor (Figure 4). In the comparison between tuberculosis and tumor, PADA in PE showed the best AUC value of $0.90(95 \% \mathrm{Cl}$ : 0.87-0.93). 
Table 3

ROC analysis of serum and pleural effusion indicators

\begin{tabular}{|lllll|}
\hline Features & AUC & Std. Error & 95\% Confidence Interval & Asymptotic Sig. \\
\hline pADA & 0.90 & 0.015 & $0.87-0.93$ & $<0.001$ \\
\hline pHsCRP & 0.79 & 0.021 & $0.75-0.83$ & $<0.001$ \\
\hline sMONp & 0.75 & 0.02 & $0.71-0.79$ & $<0.001$ \\
\hline sHsCRP & 0.73 & 0.031 & $0.67-0.79$ & $<0.001$ \\
\hline sESR & 0.71 & 0.027 & $0.66-0.76$ & $<0.001$ \\
\hline sD-dimer & 0.70 & 0.027 & $0.64-0.75$ & $<0.001$ \\
\hline
\end{tabular}

In addition, the potential combination schemes of metabolic biomarkers based on logistic regression analysis were applied to enhance the sensitivity and accuracy of diagnostic of tuberculosis patients from malignant tumor patients with PE. As shown in Figure 5, the combination of 3 markers (pADA, sMONp and sHsCRP), remarkable enhanced the AUC to 0.944 (95\% Cl: 0.925-0.964). These results indicated that 3 indicators could act as a promising combination for detection of tuberculosis from tumor with PE.

\subsection{Diagnostic indicator of pADA for evaluation in different clinical patients' characteristics}

According to the above, we observed the best indicator namely pADA was close to the value of combination of three markers (the only one $\geq 0.9$ ). Thus, we were interested in the alteration of indicators in different characters of patients. In the stratified analysis, the AUC of pADA of males was 0.897 , females were 0.910 , females was higher than males. The AUC of patients with fever was 0.932 , and the AUC of patients without fever was 0.894 , suggesting that the diagnosis accuracy of fever patients was higher. The AUC of Age $<25$ was $0.932,25 \leq$ Age $<45$ was $0.939,45 \leq$ Age $<65$ was 0.889 , and Age $\geq 65$ was 0.868. Although with the increase of age, the AUC showed a downward trend, and the diagnostic accuracy decreased (Figure 6). The indicator pADA still showed the continuous distinguishing function as a diagnostic marker for tuberculosis and tumor patients with $\mathrm{PE}$.

\section{Discussion}

In clinical practice, it is very common and critical to distinguish TPE from MPE as the pathogenesis, treatment, and recovery of the two diseases are different[18]. Thus early diagnosis is particularly important. In this study, comparing TPE with MPE, the patients of TPE were more likely to have a fever and the MPE was more bloody $(p<0.01)$. It is commonly accepted by clinician that fever is a common symptom in patients with TPE[19]. Other researchers proved that compared PE caused by malignant lymphoma, patients with TPE have a higher probability of fever (Fever $>37.5^{\circ} \mathrm{C}, \mathrm{MPE}=12 \%, \mathrm{TPE}=48 \%, p$ $<0.01$ ) [20]. Moreover, consistent with our results, other clinicians also observed that MPE is mostly 
bloody[21], which may be related to tumor invasion and destruction of capillaries leading to blood leakage[22].

The percentage of Rivalta test positive of TPE was higher than MPE in our results $(p<0.01)$. It has reported that the positive rate of the Rivalta test is parallel to the amount of total protein in body cavity effusion [23]. Some researchers showed that the protein level in TPE is higher than MPE $(p<0.05)$ [24]. Consistently, out results showed that the total protein (sTP) in TPE is higher than MPE $(69.09 \pm 1.72 \mathrm{vs}$ $64.01 \pm 0.67, p=0.15$ ) which could explain that the percentage of Rivalta test positive of TPE was higher than MPE.

In the study, the serum D-dimer were higher in the TPE group than the MPE group $(2.69 \pm 1.19 \mathrm{vs}$ $0.67 \pm 0.07, p=0.37$ ). It is proved by other researchers that the serum D-dimer level of TPE patients was higher than MPE patients[14]. However, they found the difference of D-dimer in PE was more obvious[14]. These show that D-dimer is a highly sensitive index in serum and PE, which helps to identify TPE and MPE.

We also observed the sLDH were higher in the MPE group than in the TPE group (221.54 $\pm 31.01 \mathrm{vs}$ $170.62 \pm 6.02, p=0.12)$. It has been reported that SLDH level was positively correlated with lymphomaassociated malignant PE (L-MPE) (OR: 1.005, 95\% confidence interval: 1.003-1.007, $p<0.001)$. sLDH > 460U/L distinguishes L-MPE from TPE with a sensitivity of $76 \%$ and a specificity of $81 \%[20]$. Consistent with our results, other researchers observed that the SLDH in MPE is higher than in TPE( $p=0.08)$ [25]. In multivariate logistic regression analysis, the ratio of sLDH: pleural fluid lymphocyte count (PELC) was positively correlated with MPE. The sensitivity and specificity of the ratio of sLDH: PELC were 0.63 (95\% $\mathrm{Cl} 0.51-0.73)$ and $0.85(95 \% \mathrm{Cl} 0.68-0.94)$ [25]. So sLDH is an important indicator for distinguishing TPE from MPE.

High sensitivity $\mathrm{C}$ reactive protein (HsCRP) is widely used as a sensitive but non-specific marker of systemic inflammation [26, 27]. Increased sHsCRP levels have been reported in many lung diseases, including tumors and tuberculosis $[28,29]$. In our study, the median levels of both pHsCRP and sHsCRP were both higher in the TPE group than in the MPE group ( $24.63 \pm 1.16$ vs $8.07 \pm 0.87$ and $50.30 \pm 2.67$ vs $19.25 \pm 3.20, p<0.01)$. The AUC of pHsCRP and sHsCRP were 0.79 and 0.73 . Consequently, HsCRP is an important reference indicator to differentiate TPE from MPE. A meta-analysis showed that the optimal critical value of pHsCPR was $21.9 \mathrm{mg} / \mathrm{dL}$, which values above the critical value were classified as TPE and below the critical value were classified as MPE, the sensitivity was 0.91 (0.73 to 0.98 ), and specificity was $0.82(0.7$ to 0.9$)[30]$.

Although HsCRP is a valuable diagnostic indicator, the diagnosis efficiency is low, so the choice of multiindex joint analysis is conducive to improving the diagnosis efficiency and accuracy. Through logical analysis, we had selected six relevant indicators (pADA, pHsCRP, sD-dimer, sESR, sHsCRP, and sMONp), with the Logistic regression model, 3 variables of pADA, sMONp, and sHsCRP could better help distinguish patients with PE by tuberculosis from malignant tumor. The combined AUC of the three factors can reach 0.94 (95\% Cl: $0.91 \sim 0.97)$, higher than any single index, which has great significance 
for the clinical differentiation between TPE and MPE. Agreed with our data, there is a study analyzed 118 patients, including 84 patients with MPE (71.2\%) and 34 patients with TPE (28.8\%). They also found the pADA of TPE is higher than MPE $(p<0.05)$ [25]. Moreover, others have proved that the elevated levels of sHsCRP and pADA in PE were useful in distinguishing TPE from MPE[31]. However, there is one study with a different result. After analyzing 17 patients with L-MPE and 216 patients with TPE. They found there was no statistically significant difference in sHsCRP and pADA levels between the two groups[20] which could be related to number of MPE patients included in the study.

At present, to achieve better treatment efficacy in clinical practice, many researchers were interested in exploring the differentiation between TPE and MPE [32-34]. Some of them were focused on the inflammatory factors. It has been reported that the biomarkers of PE in 22 patients with MPE and 5 patients with TPE were compared. IL-1, IP-10, IL-13 and IFN- $\gamma$ were significantly higher in TPE $(p<0.05)$. The level of basic fibroblast growth factor in MPE was higher than that in TPE $(p<0.05)$ [33]. The highest AUC is IP-10 (AUC =0.95, 95\% confidence interval, $p<0.01)$, followed by IL-13 (AUC =0.86, 95\% confidence interval, $p<0.05$ ) [33]. However, though one of the indicators in this study has a high value of AUC, the detection is not a common clinical indicator, and the detection is complicated. And the sample size is small, the reliability is weak, and it is difficult to perform stratified analysis. Another study found that Fibronectin (FN) and cathepsin G (CTSG) in patients with MPE were significantly higher than in patients with TPE, while the leukotriene-a4 hydrolase (LTA4H) was lower than in patients with TPE[34]. The AUC value was determined to be 0.285 for $\mathrm{FN}$ (95\% Cl: $0.174-0.396), 0.64$ for LTA4H (95\% Cl: 0.518-0.762), 0.337 for CTSG (95\% Cl: $0.218-0.456)$, and 0.793 for a combination of these candidate markers $(95 \% \mathrm{Cl}$, 0.697-0.888). The AUC value is significantly lower than in our study.

In this study, our results has more significant advantages of high diagnostic accuracy (high AUC value, high sensitivity, and specificity) and large sample size which mean high data reliability. More favorably, pADA, sHsCRP and sMONp are all clinically common and easy-to-collect specimens, which are convenient and cheap to test, and will not increase the additional burden on patients. Because of the large sample size, hierarchical analysis can be performed and it is found that the diagnostic efficiency of pADA is different in various age groups, and as the age increases, the diagnostic efficiency of pADA gradually decreases. This phenomenon could be related to the percentage of tuberculosis decreased while the cancer diagnosis increased with the age growth. It suggests that the patients under 45 years old could choose the single indicator of pADA for diagnostic detection.

Of course, the gold standard for differentiating TPE and MPE in clinical practice still relies on pathological tissue biopsy[32], all cases in this study were examined by thoracoscopy, and pathological biopsy was completed in most cases, which ensured the accuracy of the diagnosis of the patients, but for some patients who refuse to accept the invasive examination, or whose constitution is difficult to bear invasive examination, the effective detection index of non-invasive provides a strong basis for timely diagnosis and accurate treatment. It is worth doing further research and exploration.

\section{Conclusion}


In summary, our results show that we have found some non-invasive and valuable markers for differentiating TPE from MPE. Although the gold standard for differentiating TPE and MPE still relies on pathological tissue biopsy, but for some patients who refuse to accept the invasive examination, or whose constitution is difficult to bear invasive examination, the effective detection index of non-invasive provides a strong basis for timely diagnosis and accurate treatment.

\section{Abbreviations}

AUC, area under curve; CA, cancer; CTSG, cathepsin G; FN, Fibronectin; HsCRP, high sensitivity C reactive protein; LDH, lactate dehydrogenase; LTA4H, leukotriene-a4 hydrolase; L-MPE, lymphoma-associated malignant pleural effusions; MPE, malignant pleural effusion; PE, pleural effusion; PELC, pleural fluid lymphocyte count; pADA, hydrothorax adenosine dehydrogenase; pAMS, hydrothorax amylase; pCell, hydrothorax cells; $\mathrm{pGLU}$, hydrothorax glucose; $\mathrm{pHsCRP}$, hydrothorax high sensitivity $\mathrm{C}$ reactive protein; pMON, hydrothorax monocytes; pNC, hydrothorax nucleated cells; pTC, hydrothorax total cholesterol; pTP, hydrothorax total protein; sAlb, blood albumin; SALP, blood alkaline phosphatase; sALT, alanine aminotransferase; sAPTT, activated partial prothrombin time; sAST, aspartate aminotransferase; $\mathrm{SA} / \mathrm{G}$, blood A/G; sBASp, percentage of blood basophil cells; sCa, serum calcium; sCK, blood creatine kinase; sCKI, blood creatine kinase isoenzyme; SCL, blood chlorine; SCRE, serum creatinine; sD-dimer, blood D-

dimer; sEOSp, percentage of eosinophil; sESR, erythrocyte sedimentation rate; sFbg, blood fibrin; sFDP, fibrinogen degradation products; sGLO, globin; sGRANp, percentage of blood granulocytes; sHCO3; , blood bicarbonate; SHGB, hemoglobin; SHsCRP, blood high sensitivity $\mathrm{C}$ reactive protein; sINR, blood internationalization standardized ratio; sK, Serum kalium; sLDH, serum lactate dehydrogenase; sLYMp, lymphocyte percentage; sMg, serum magnesium; sMONp, percentage of blood monocyte; sNA, blood natrium; sP, serum phosphate; SPA, blood prealbumin; sPLT, platelet; sPT, prothrombin time; SPTA, prothrombin activity; sRBC, red blood cell; sPTR, prothrombin time ratio; sTB, total bilirubin; sTBA, total bile acid; STP, blood total protein; STT, blood thrombin time; sUREA, blood urea; sWBC, white blood cell; saHDH, blood a hydroxybutyrate dehydrogenase; sY-GT, blood y glutamyl transpeptidase; TB, tuberculosis; TPE, tuberculous pleural effusion.

\section{Declarations}

\section{Ethics approval and consent to participate}

The study received approval from ethics committee of Shiyan Taihe Hospital. All patients agreed to participate in the study and signed informed consent forms.

\section{Consent for publication}

Not applicable.

\section{Availability of data and materials}


The datasets used and/or analysed during the current study are available from the corresponding author on reasonable request.

\section{Competing interests}

The authors declare no competing financial interests.

\section{Funding}

This work was supported by Macao Science and Technology Development Fund (Project no: 0096/2018/A3, 001/2020/ALC) and the NSFC overseas and Hong Kong and Macao Scholars Cooperative Research Fund Project (Project no: 81828013) and the 2020 Guangdong Provincial Science and Technology Innovation Strategy Special Fund (Guangdong-Hong Kong-Macau Joint Lab) (No: 2020B1212030006) and Taihe Hospital Fund Project (Project no: 2019JJXM007). This work was supported by grants from Dr. Neher's Biophysics Laboratory for Innovative Drug Discovery \001/2020/ALC凶.

\section{Authors' contributions}

(I) Conception and design: ELHL, RZL, MFW, XJY; (II) Administrative support: LL, YJT; (III) Provision of study materials or patients: MFW, TR, ZXF, XXF, HDP; (IV) Collection and assembly of data: MFW; (V) Data analysis and interpretation: RZL, WYM, JW, IK; (VI) Manuscript writing: All authors; (VII) Final approval of manuscript: All authors.

\section{Acknowledgements}

Not applicable.

\section{References}

1. Zhang F, Wang J, Zheng X, Hu L, Chen J, Jiang F, Wang Y: Clinical value of jointly detection pleural fluid Midkine, pleural fluid adenosine deaminase, and pleural fluid carbohydrate antigen 125 in the identification of nonsmall cell lung cancer-associated malignant pleural effusion. Journal of clinical laboratory analysis 2018, 32(8):e22576.

2. Garske LA, Kunarajah K, Zimmerman PV, Adams L, Stewart IB: In patients with unilateral pleural effusion, restricted lung inflation is the principal predictor of increased dyspnoea. PloS one 2018, 13(10):e0202621.

3. Doelken P: Clinical implications of unexpandable lung due to pleural disease. The American journal of the medical sciences 2008, 335(1):21-25.

4. Sahn S, Huggins J, San José M, Alvarez-Dobano J, Valdes L: Can tuberculous pleural effusions be diagnosed by pleural fluid analysis alone? The International journal of tuberculosis and lung disease 2013, 17(6):787-793. 
5. Lange C, Mori T: Advances in the diagnosis of tuberculosis. Respirology 2010, 15(2):220-240.

6. Porcel JM, Gasol A, Bielsa S, Civit C, Light RW, Salud A: Clinical features and survival of lung cancer patients with pleural effusions. Respirology 2015, 20(4):654-659.

7. McGrath EE, Warriner D, Anderson PB: Pleural fluid characteristics of tuberculous pleural effusions. Heart \& Lung 2010, 39(6):540-543.

8. Maskell N, Butland R: Pleural Diseases Group, Standards of Care Committee, British Thoracic Society. BTS guidelines for the investigation of a unilateral pleural effusion in adults. Thorax 2003, 58(Suppl. 2):ii8-ii17.

9. Gao B-A, Zhou G, Guan L, Zhang L-Y, Xiang G-M: Effectiveness and safety of diagnostic flexi-rigid thoracoscopy in differentiating exudative pleural effusion of unknown etiology: a retrospective study of 215 patients. Journal of thoracic disease 2014, 6(5):438.

10. Zhang M, Li D, Hu Z-D, Huang Y-L: The diagnostic utility of pleural markers for tuberculosis pleural effusion. 2019, 8(9):607.

11. Helmy NA, Eissa SA, Masoud HH, Elessawy AF, Ahmed Rl: Diagnostic value of adenosine deaminase in tuberculous and malignant pleural effusion. Egyptian Journal of Chest Diseases and Tuberculosis 2012, 61(4):413-417.

12. Goto M, Noguchi Y, Koyama H, Hira K, Shimbo T, Fukui T: Diagnostic value of adenosine deaminase in tuberculous pleural effusion: a meta-analysis. Annals of clinical biochemistry 2003, 40(4):374381.

13. Samanta S, Sharma A, Das B, Mallick AK, Kumar A: Significance of total protein, albumin, globulin, serum effusion albumin gradient and LDH in the differential diagnosis of pleural effusion secondary to tuberculosis and cancer. Journal of clinical and diagnostic research: JCDR 2016, 10(8):BC14.

14. Shen Y, Yang T, Jia L, Wang T, Chen L, Wan C, Wang L, Yan Y, Yi Q: A potential role for D-dimer in the diagnosis of tuberculous pleural effusion. Age 2013, 17:201-205.

15. Antonangelo L, Vargas FS, Seiscento M, Bombarda S, Teixera L, Sales RKBd: Clinical and laboratory parameters in the differential diagnosis of pleural effusion secondary to tuberculosis or cancer. Clinics 2007, 62(5):585-590.

16. Chen M-L, Yu W-C, Lam C-W, Au K-M, Kong F-Y, Chan AY-W: Diagnostic value of pleural fluid adenosine deaminase activity in tuberculous pleurisy. Clin Chim Acta 2004, 341(1-2):101-107.

17. Radjenovic-Petkovic T, Pejcic T, Nastasijevic-Borovac D, Rancic M, Radojkovic D, Radojkovic M, Djordjevic l: Diagnostic value of CEA in pleural fluid for differential diagnosis of benign and malign pleural effusion. Med Arh 2009, 63(3):141-142.

18. Jin D, Chen Y, Wang Z, Wang S, Bunjhoo H, Zhu J, Cao Y, Xiong W, Xiong S, Xu Y: Diagnostic value of interleukin 22 and carcinoembryonic antigen in tuberculous and malignant pleural effusions. Experimental and therapeutic medicine 2011, 2(6):1205-1209.

19. Zhai K, Lu Y, Shi H-Z: Tuberculous pleural effusion. Journal of thoracic disease 2016, 8(7):E486. 
20. Kim CH, Oh HG, Lee SY, Lim JK, Lee YH, Seo H, Yoo SS, Lee SY, Cha SI, Park JY: Differential diagnosis between lymphoma-associated malignant pleural effusion and tuberculous pleural effusion. Annals of translational medicine $2019,7(16): 373$.

21. Karkhanis VS, Joshi JM: Pleural effusion: diagnosis, treatment, and management. Open access emergency medicine: OAEM 2012, 4:31-52.

22. Chikileva IO, Anisimova NY, Shubina IZ, Kiselevsky MV: Pathogenesis of Malignant Effusions. In: Malignant Effusions. edn.: Springer; 2012: 11-21.

23. Zhu S, Du L, Xu D, Lu Z, Xu T, Li J, Xu K, Ye J, Song Y: Ascitic fluid total protein, a useful marker in non-portal hypertensive ascites. J Gastroenterol Hepatol 2020, 35(2):271-277.

24. Umar M, Iqbal Z, Basit A, Khan MY, Javaid A: Validity of pleural fluid protein in differentiating tuberculous from malignant pleural effusion. Pakistan Journal of Chest Medicine 2018, 24(3):141146.

25. Verma A, Dagaonkar RS, Marshall D, Abisheganaden J, Light R: Differentiating malignant from tubercular pleural effusion by cancer ratio plus (cancer ratio: pleural lymphocyte count). Canadian respiratory journal 2016, 2016:7348239.

26. Park D-S, Kim D, Hwang K-E, Hwang Y-R, Park C, Seol C-H, Cho K-H, Kim B-R, Park S-H, Jeong E-T et al: Diagnostic value and prognostic significance of pleural C-reactive protein in lung cancer patients with malignant pleural effusions. Yonsei Med J 2013, 54(2):396-402.

27. Pepys MB, Hirschfield GM: C-reactive protein: a critical update. The Journal of clinical investigation 2003, 111(12):1805-1812.

28. Allin $\mathrm{KH}$, Nordestgaard BG: Elevated C-reactive protein in the diagnosis, prognosis, and cause of cancer. Crit Rev Clin Lab Sci 2011, 48(4):155-170.

29. Yoon C, Chaisson LH, Patel SM, Allen IE, Drain PK, Wilson D, Cattamanchi A: Diagnostic accuracy of C-reactive protein for active pulmonary tuberculosis: a meta-analysis. Int J Tuberc Lung Dis 2017, 21(9):1013-1019.

30. Angeles RRB, King RE, Benedicto J, Rivera A: Accuracy of pleural fluid c-reactive protein in differentiating between tuberculous and malignant pleural effusions: a meta-analysis. In., vol. 54: Eur Respiratory Soc; 2019: PA2978.

31. Lee J, Lee YD, Lim JK, Lee DH, Yoo SS, Lee SY, Cha SI, Park JY, Kim CH: Predictive factors and treatment outcomes of tuberculous pleural effusion in patients with cancer and pleural effusion. The American journal of the medical sciences 2017, 354(2):125-130.

32. Liu Q, Yu Y-X, Wang X-J, Wang Z, Wang Z: Diagnostic Accuracy of Interleukin-27 between Tuberculous Pleural Effusion and Malignant Pleural Effusion: A Meta-Analysis. Respiration 2018, 95(6):469-477.

33. Chen K-Y, Feng P-H, Chang C-C, Chen T-T, Chuang H-C, Lee C-N, Su C-L, Lin L-Y, Lee K-Y: Novel biomarker analysis of pleural effusion enhances differentiation of tuberculous from malignant pleural effusion. International journal of general medicine 2016, 9:183. 
34. Shi J, Li P, Zhou L, Qi S, Wang B, Li D, Duan L, Chen WX, Xia J, Zou L: Potential biomarkers for antidiastole of tuberculous and malignant pleural effusion by proteome analysis. Biomarkers in medicine 2019, 13(02):123-133.

\section{Figures}

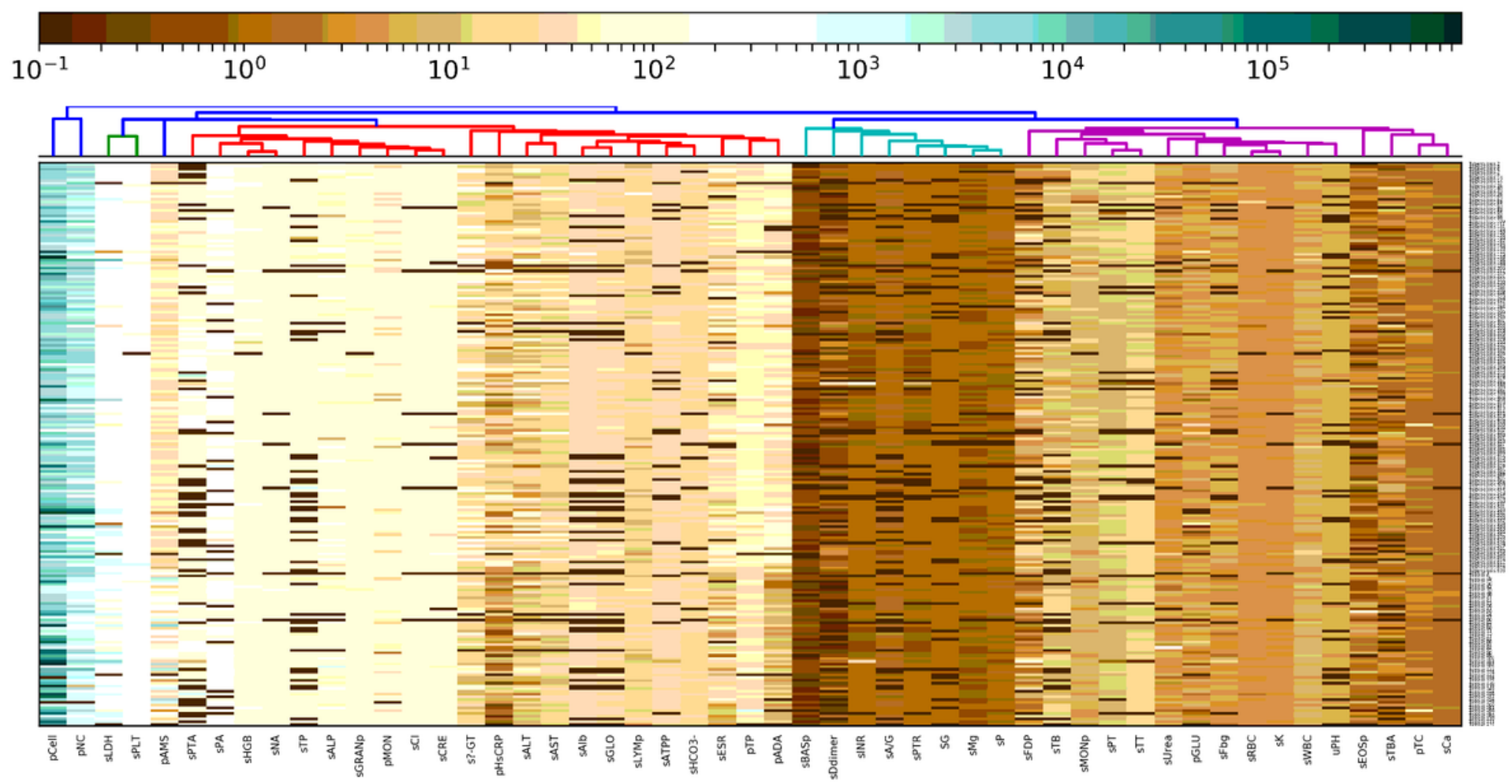

\section{Figure 1}

Tuberculosis and tumor patients were grouped by unsupervised hierarchical clustering of serum and pleural effusion clinical indicators 

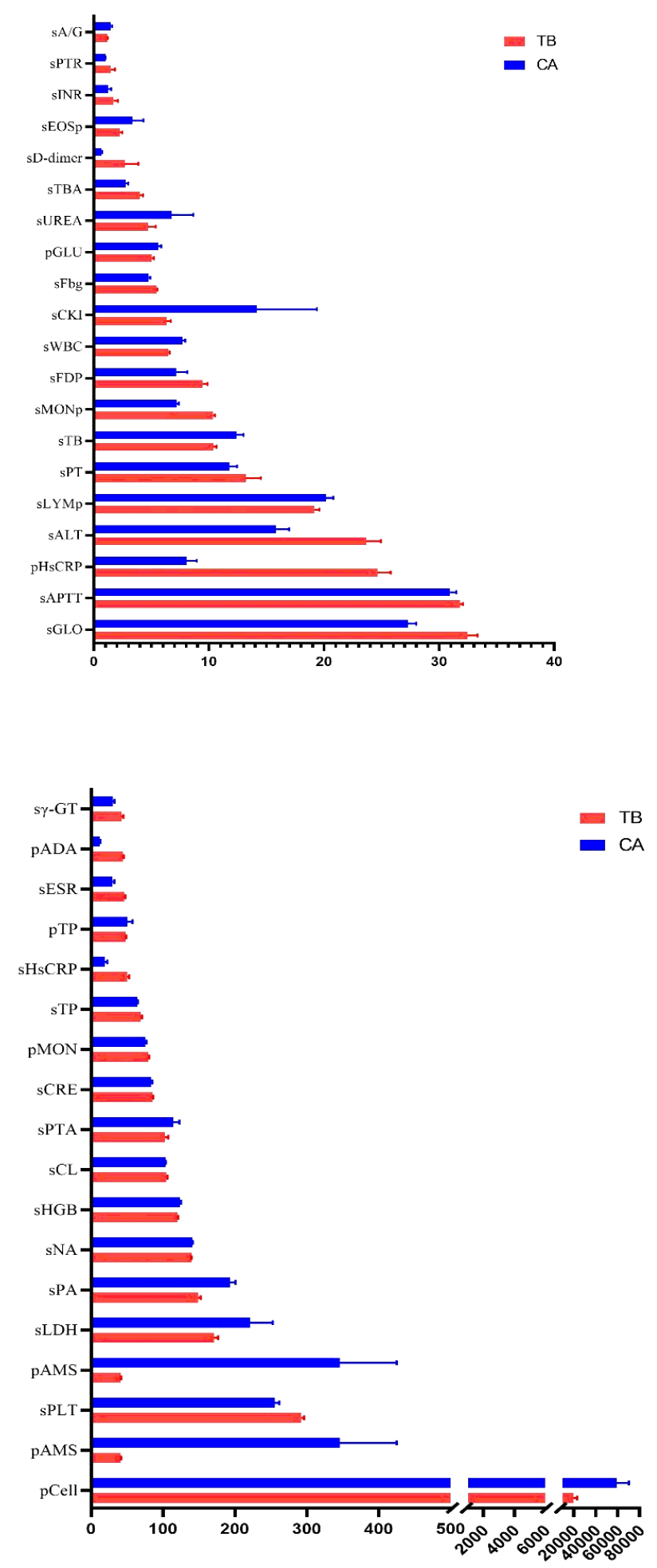

\section{Figure 2}

Serum and pleural effusion clinical indicators $(\mathrm{N}=37)$ with statistical significance of tuberculosis and tumor (mean value with SD) 
(A)

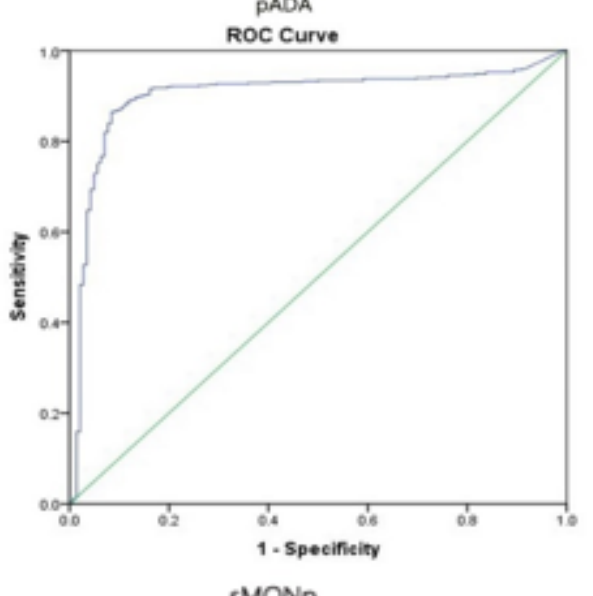

(C)

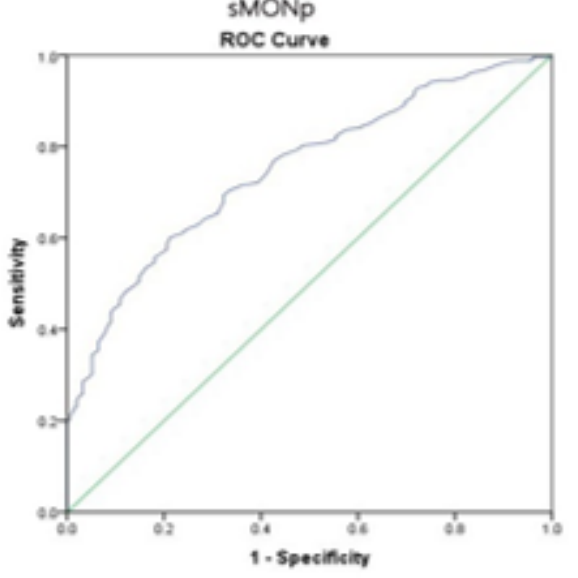

(E)

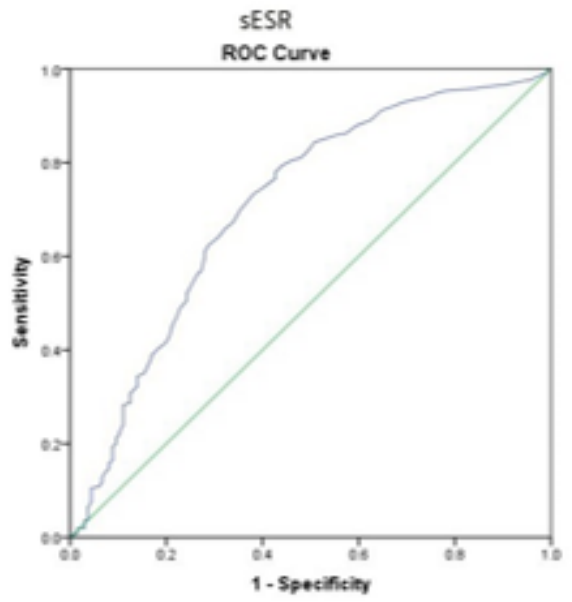

(B)

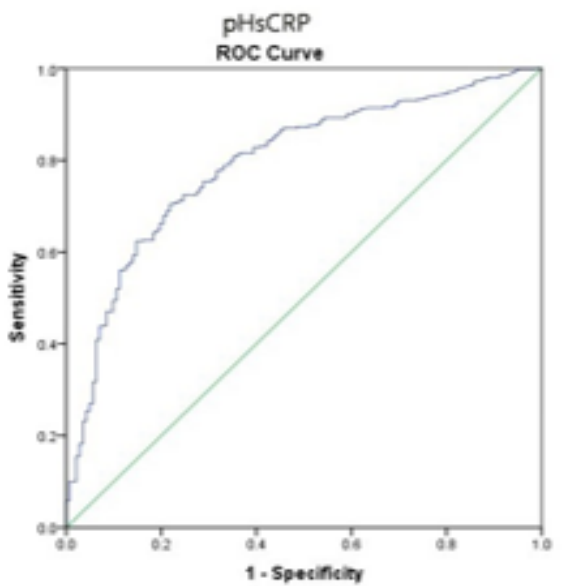

(D)

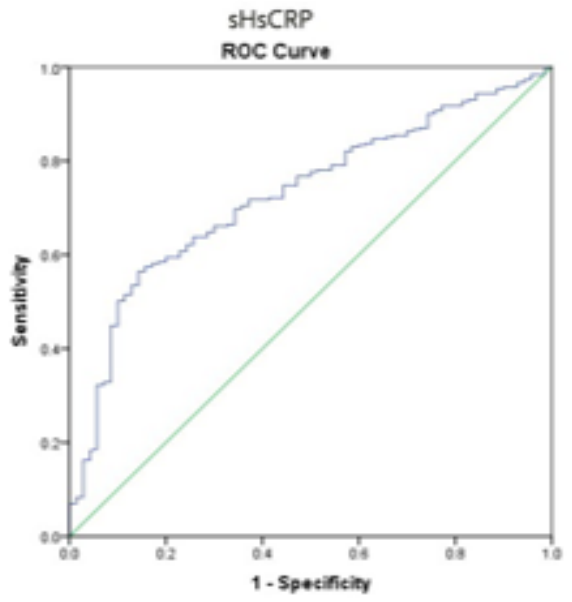

(F)

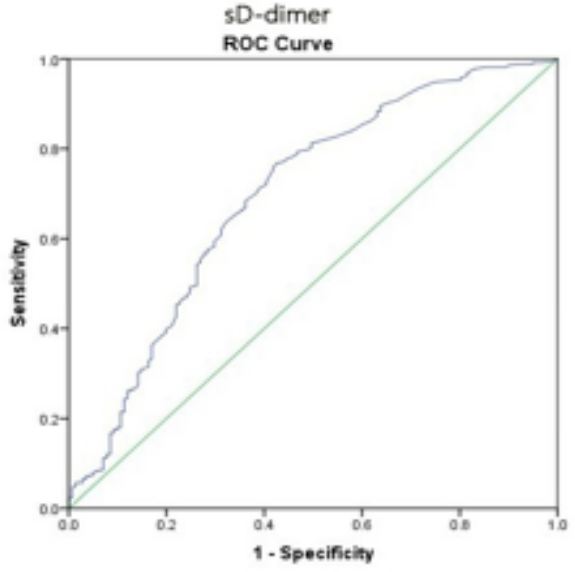

\section{Figure 3}

ROC analysis of serum and pleural effusion clinical indicators distinguishing tuberculosis from tumor patients $(A U C>0.7)$ 


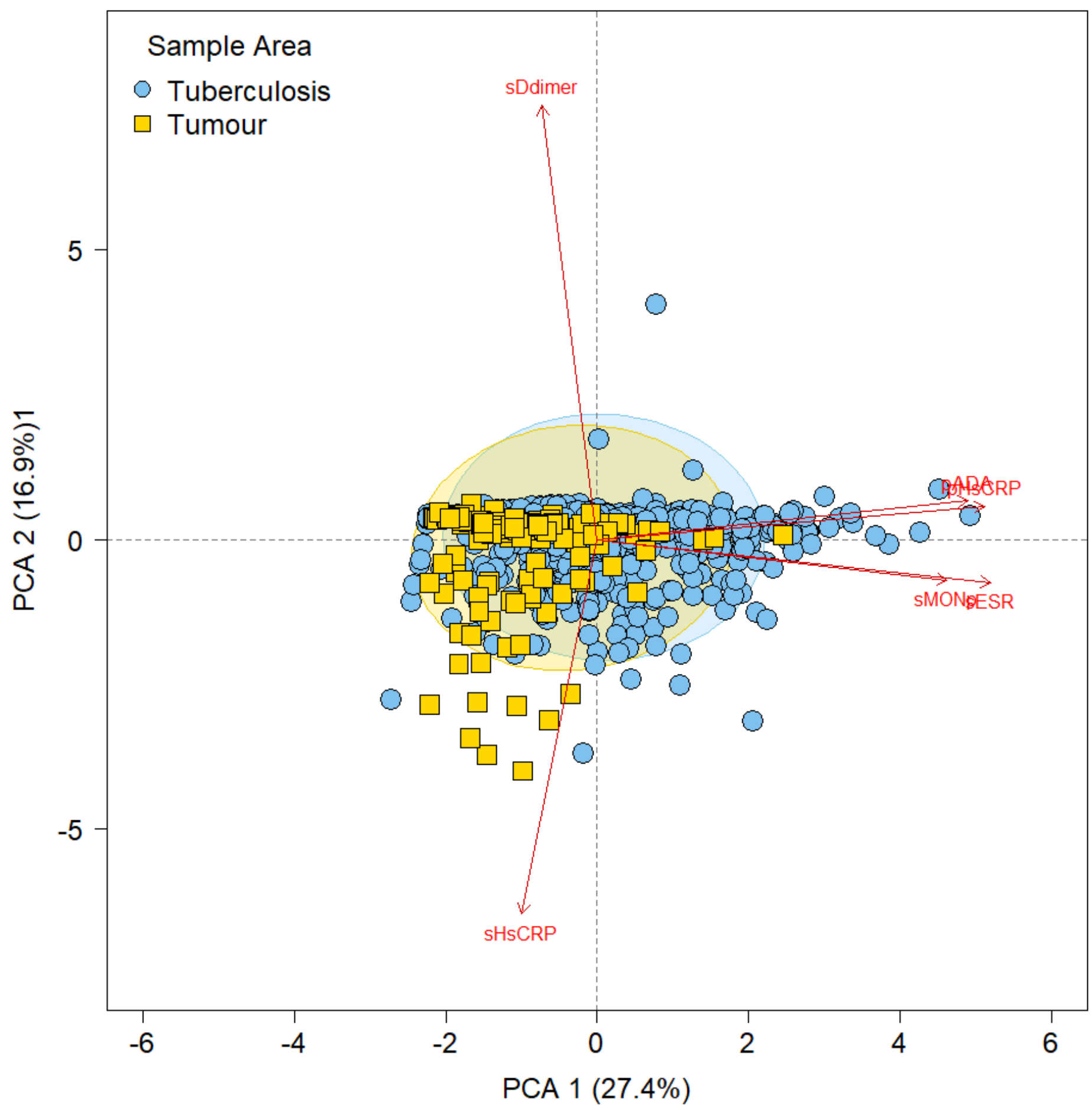

Figure 4

PCA analysis of 6 comparable serum and pleural effusion indicators of tuberculosis and tumor patients 


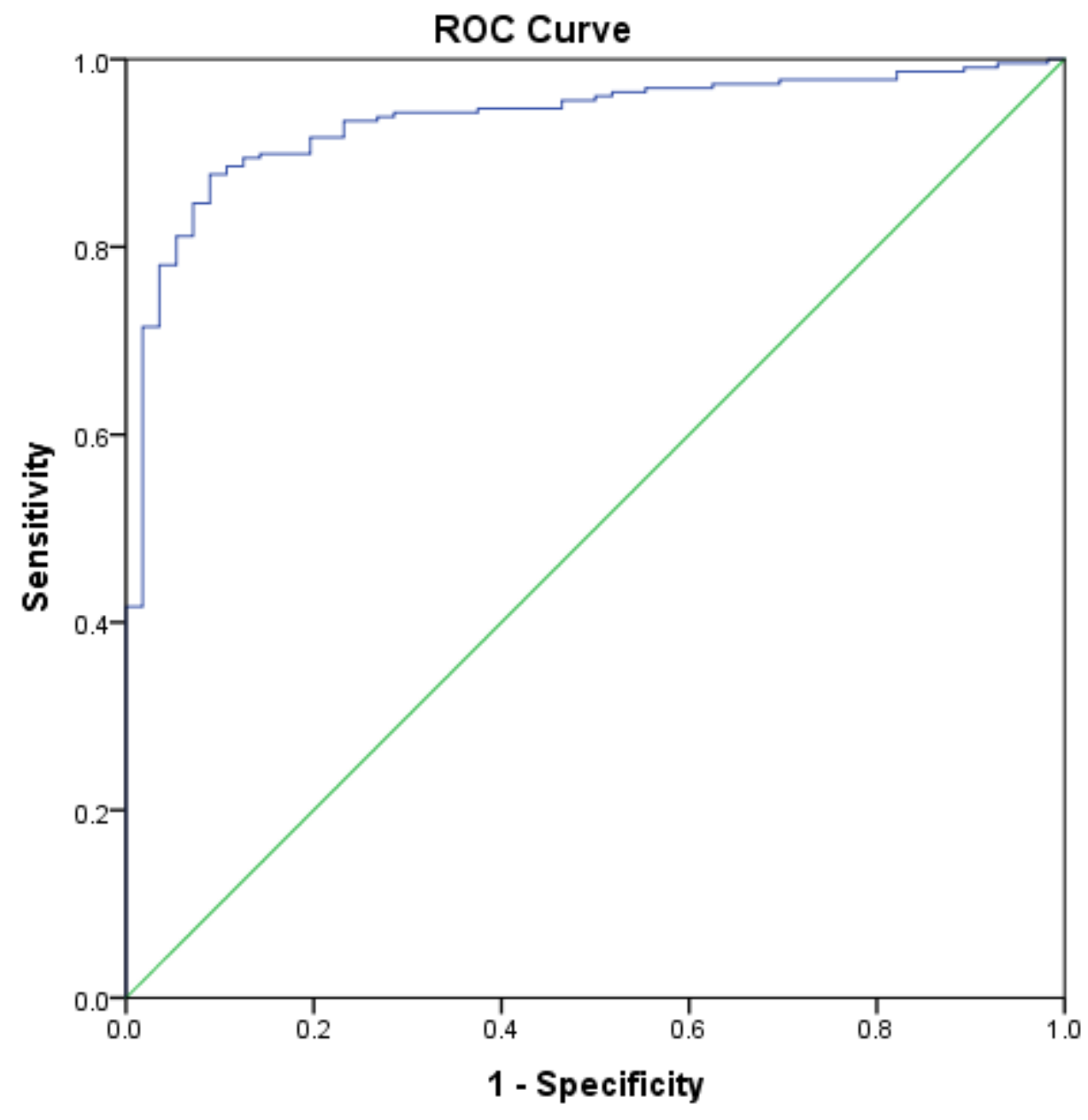

Figure 5

ROC analysis of combined indicators in tuberculosis detection form tumor patients 
(A)

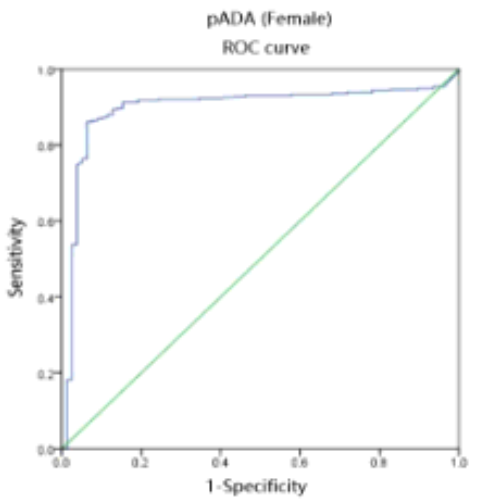

(C)

(E)

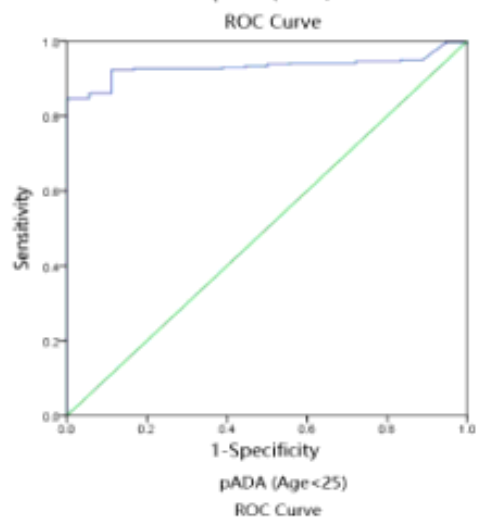

(G)
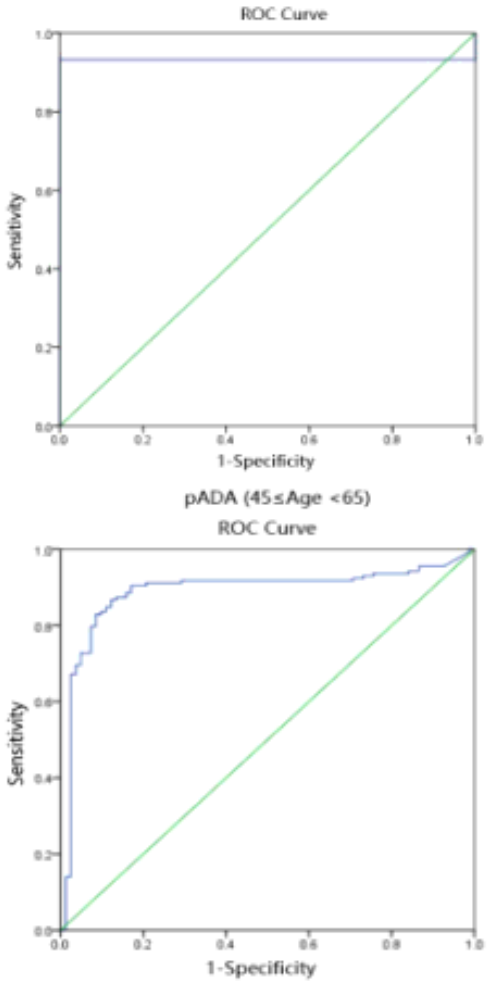

(B)

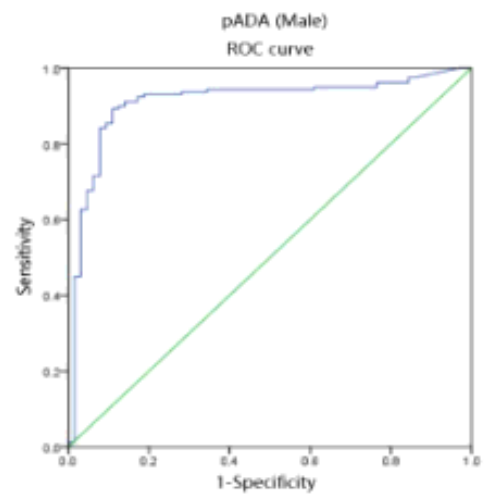

(D)

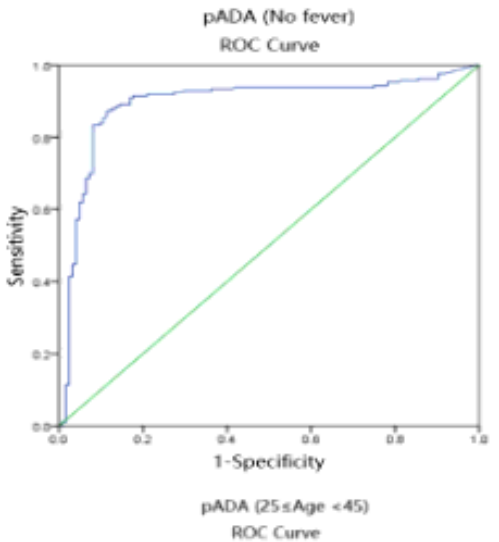

(F)

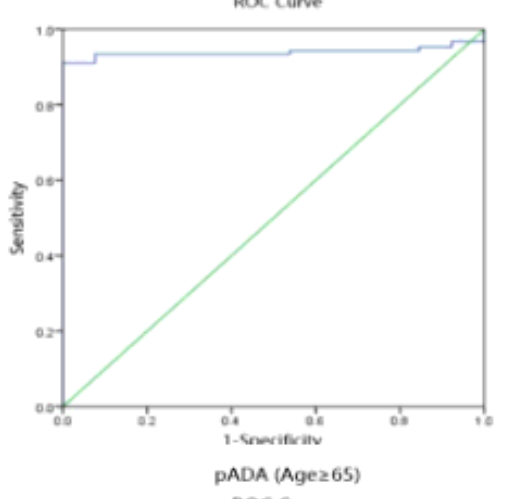

(H)

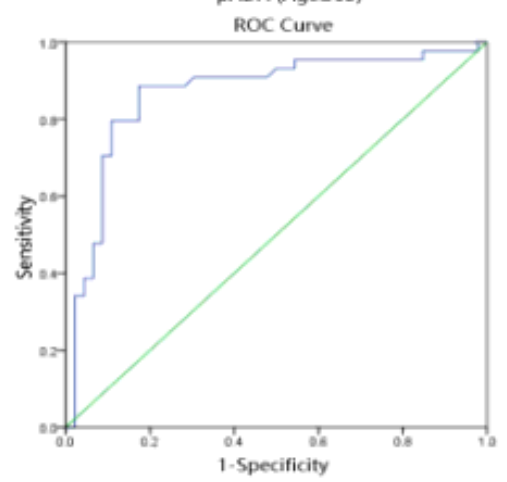

Figure 6

ROC analysis of selected indicator PADA in different status of patients 